\title{
Multi-criteria and Hierarchical Level Energy Management System for Light Solar Vehicle Integrating a Supercapacitor
}

\author{
Abdellatif Belattar $^{1}$, Achour El Hamdaouy ${ }^{2 *}$, Abdessalam Ait Madi ${ }^{1}$ \\ ${ }^{1}$ National School of Applied Sciences, Ibn Tofail University, B.P. 242, Kenitra 14090, Morocco \\ ${ }^{2}$ High School of Technology, Ibn Tofail University, B.P. 242, Kenitra 14090, Morocco
}

Corresponding Author Email: a.elhamdaouy@uit.ac.ma

https://doi.org/10.18280/jesa.530302

Received: 12 April 2020

Accepted: 30 May 2020

\section{Keywords:}

light solar vehicle, photovoltaic system, battery, supercapacitor, energy management, multi-criteria, hierarchical level, fuzzy logic

\begin{abstract}
In this paper, multi-criteria and hierarchical energy management system (EMS) for a light solar vehicle (LSV) is investigated. This vehicle uses of a lithium-ion battery (bat), a supercapacitor (SC), a photovoltaic (PV) generator and a propulsion system. The proposed management strategy aims to enhance power sharing between multiple sources with different characteristics, and to optimize power trajectory in order to avoid the energy losses in the DC/DC converters. For this sake, the developed strategy is based on five modes switching process. The proposed EMS consists of three hierarchical levels. The high level defines dynamically mode operation according to system situation and management strategy (decision strategy). It employs the state of charge of the battery (SOCbat), the state of charge of SC (SOCsc), the PV power (Ppv) and the load power (Pload) as a four-information sensor to distinguish different modes and determine the mode selected. While the medium level, integrates an algorithm to compute the reference power for the SC and the battery at each instant and according to selected mode. The low level plays an interface between EMS and DC/DC converters. It is composed from the proportional integral (PI) controllers which provide a pulse width modulation (PWM) signal to DC/DC converters. It is noteworthy here that the fuzzy logic (FL) technology is used to implement the suggested EMS. The obtained results of various simulations demonstrate the effectiveness and reliability of the proposed strategy.
\end{abstract}

\section{INTRODUCTION}

The demand of petroleum products is increasing day after day. This is due to unavoidable need for this vital energy, its serious depletion and aberrant usage, besides the conflicts in the main producing areas. Consequently, the price of these products is increasing more and more. Thus, obtaining other kinds of energy sources has become an indisputable necessity. For this reason, the use of renewable energy has been attracted the attention of the academic and industrial communities around the world, particularly solar energy source which has become a promising energy source widely used in various fields [1]; including vehicles field which has already started using solar energy source as a clean renewable [2]. No doubt, the principle advantage of the integration of solar energy in this field is the limitation of toxic gases emissions [3]. Moreover, several race vehicles that are operated by solar energy are taking place, more and more, in many countries every year. The Moroccan country is also one of them.

In LSV used in these race vehicles, the PV generator is the main source in the power system and as its production depends on sunlight [4], a storage system is required to ensure the energy demand all time $[5,6]$. In order to achieve the same power and the same energy density as in internal combustion engines, the battery is combined with a SC which can provide high power during acceleration periods or under high demand. Indeed, Gagliardi et al. [7] have verified the possibility of integrating SC and batteries in a vehicle taking into account experimental tests to restore the braking energy, impulse currents and minimizing battery intervention. Pay et al. [8] have also proven that the combination of the battery and the $\mathrm{SC}$ can reduce the maximum current in the battery of $40 \%$.

The control and the management strategy for multi-source system are very crucial in coordinating different sources involved and in enhancing vehicle's dynamic and static performances [9-15]. Hannan et al. [16] have proposed a digital controller that acts as an EMS for light electric vehicle (EV) containing a battery, a SC and a fuel cell. By his turn, Ahmed et al. [17] have developed a real time EMS based on a system of fuzzy inference rules optimized through a genetic algorithm (GA) for a multi-source system in EV. Particularly, a description of an EMS for solar race cars has been presented by Ustun et al. [18]. In these works, any guidance is provided for best operating range for SOCsc and/or SOCbat.

The decomposition of energy management and control in multi-level has been discussed in other works [19, 20]. In fact, Joao et al. [19] have developed a multi-level EMS integrating the rule based meta-heuristic approach to define the assignment factors of the SC and the battery. However, integrating the PV generator in the power system can make important changes in energy management strategy. Leon et al. [20] have also presented a modular approach to design and implement a power and an EMS for EV containing a battery and a SC. Though, a study [21] presents a scheme to achieve an EMS for different vehicles in general way, still, the diversity of hybrid EVs must be taken into account. Besides, 
none of these works has considered, in their management strategies, the optimization of the power trajectory in order to avoid losses in converters.

The previous studies propose several EMS for hybrid EV. However, efforts for integrating and using the SC with PV system in hybrid light EVs are not widely implemented. Indeed, using SC along with battery might be the most important point in increasing competency of the LSV, which can be achieved by accurate and reliable power control and energy management. Furthermore, combining multiple sources into one system presents challenges related to the nature of each source, as well as optimizing power trajectory to reduce losses in converters. These challenges which motivate the authors to propose a powerful EMS that can hence energy efficiency in the vehicle as a whole.

This paper proposes a hierarchical EMS for an LSV equipped with three energy sources: PV generator, battery and SC. The developed management strategy provides a good power sharing between sources taking into consideration their complementarily and constraints. The EMS meets many criteria like optimizing, at each instant, the power trajectory to avoid maximum power losses in DC/DC converters, ensuring the best operating range for the SOCbat, feeding continuously the powertrain request and giving guidance for suitable operating range for SOCsc.

To achieve these objectives, the vehicle operation is divided into five different modes that illustrate several situations. The action of the proposed EMS on the power system occurs through three hierarchical levels: the high level which determines the required mode to operate, the medium level which implements an algorithm to compute the reference power for the SC and the battery and the third one is the low level which integrates the DC/DC converters, as an interface between the EMS and power system, to achieve optimal performances. In fact, the fuzzy technology, performed in this work, is widely used to enhance EMS in EVs [20, 22].

The present paper is organized as follows: the second section deals with the energy management constraints, the third section describes the different elements of the solar race vehicle, the fourth section investigates the proposed approach for energy management strategy, the fifth section discusses the simulation results and the sixth section concludes our paper.

\section{ENERGY MANAGEMENT FORMULATION}

CONSTRAINTS

In the LSV, the PV generator is the main source while the battery ensures the power balance between $\mathrm{PV}$ and powertrain request. The use of the SCs allows achieving good energy efficiency, high dynamic vehicle performance and lower cost.

The power flow in a multi-source system containing PV generator, battery and SC can be expressed by Eq. (1):

$$
P_{\text {dem }}(t)=P_{p v}(t)+\sum_{i=\{b a t, S C\}} P_{i}(t) \quad \forall t
$$

where, $P_{\text {dem }}(t)$ is the powertrain demand, $\mathrm{P}_{\mathrm{pv}}(\mathrm{t})$ is the $\mathrm{PV}$ power and $P_{i}(t)$ is the power of the source $I$, with $I=\{b a t, S C\}$.

The best solution of (1) is to find, at each instant, the power quantity that every source must provide to the load. This is based on the correct definition of constraints of each source especially those maximum and minimum powers for the battery and SC; it can be resumed by Eq. (2):

$$
\left\{\begin{array}{c}
P p v=P_{p v}^{\max } ; P_{p v} \geq 0 \\
P_{i}^{\text {min }} \leq P_{i} \leq P_{i}^{\text {max }} ; i=\{b a t, s c\} \\
P_{i}^{\text {min }} \leq 0 \leq P_{i}^{\text {max }}
\end{array}\right.
$$

with $P_{p v}^{\max }$ is the PV maximum power, $P_{i}^{\min }$ and $P_{i}^{\max }$ are respectively the minimum and the maximum power of source i.

To overcome the SC low energy density [9], the EMS must define dynamically a best way to maintain quickly the SOCsc at an appropriate level for any possible request.

The DC/DC converters are considered as key elements in the energy management, while the power efficiency is the major inconvenient of these elements [22,23]. That means that the power losses increase when the power goes by several converters. Thus, The EMS should optimize, as much as possible, the power trajectory in order to avoid some power losses and then improving the energy efficiency in vehicle.

Therefore, the main objective of our management strategy is continuously feeding the powertrain request with the available energy in the sources by transferring the response to high peak power to the SC and respecting the power/energy source and energy efficiency constraints. By doing so, the EMS must dynamically redefine the operation mode according to vehicle situation by providing the reference power for both $\mathrm{SC}$ and battery.

\section{SOLAR VEHICLE DESCRIPTION}

The solar vehicle considered in this study conforms to standards required by "Moroccan solar race challenge committee" [24]. As seen in the Figure 1, the solar vehicle architecture can be subdivided into three parts: energy sources, DC machine and propulsion system.

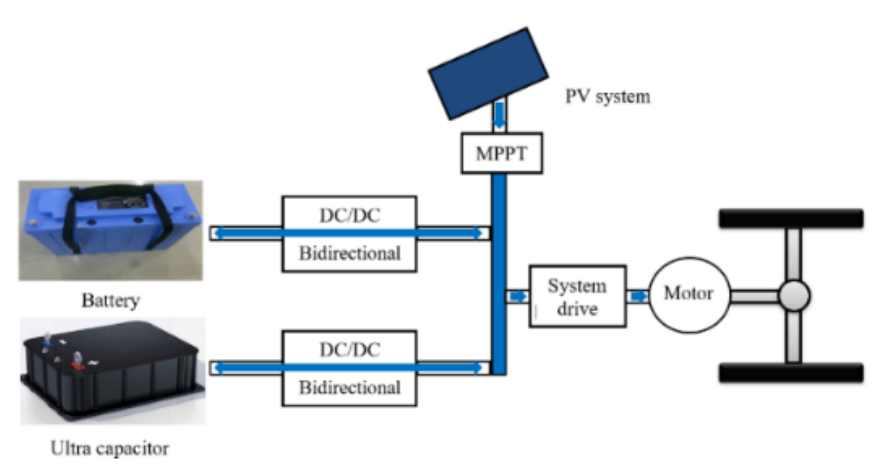

Figure 1. Solar vehicle architecture

\subsection{Photovoltaic model}

Photovoltaic technology consists to convert sunlight directly to a current. The photovoltaic module is a nonlinear device which can be modelled by a current source. The current generated by a PV module, neglecting the internal series resistance, is given by the Eq. (3) [25, 26]:

$$
I_{P V}=I_{g}-N_{p} I_{s a t}\left[\exp \left(\frac{q V_{p v}}{A K T_{m}}\right)-1\right]-I_{r s h}
$$

where, $I_{P V}$ and $V_{p v}$ are respectively the output current and output voltage of the PV module, $I_{g}$ is the generated current under a given sunlight, $I_{\text {sat }}$ is the reverse saturation current, $\mathrm{q}$ 
is the charge of an electron, $\mathrm{K}$ is the Boltzmann's constant, A is the ideality factor, $T_{m}$ is the PV module temperature $(\mathrm{K})$, and $I_{r s h}$ is the current due to intrinsic shunt resistance.

The PV system in our vehicle consists of four modules of 250W. Each module is associated with a DC/DC boost converter which is controlled by the maximum power point (MPP) tracking algorithm perturb \& observe to extract the available maximum power.

\subsection{Battery model}

Lithium-ion is the most popular type of batteries used in EVs, they have the advantage to provide a high energy density with low weight. This battery type is modelled by a dynamic lithium-ion model proposed by Trambloy et al. [27]. This battery model is simulated by a variable voltage source in series with an internal resistance. The characteristic equation of this battery is given by the Eq. (4):

$$
U_{b a t}=E_{b a t}-R_{i} \cdot i
$$

where, $U_{b a t}$ is the battery voltage, $E_{b a t}$ is open circuit voltage, i is the battery current and $\mathrm{R}_{\mathrm{i}}$ is the internal resistance which is assumed to be constant during the charge and the discharge cycles, and which does not vary with the amplitude of the current.

\subsection{SC model}

The modelling of the electrical behavior of the SC is similar to that of a simple capacitor; its capacity $\mathrm{C}$ depends on the surface $S$ of the two parallel plates, the distance $\mathrm{d}$ between them, and the permittivity of the dielectric material $\varepsilon_{0} \varepsilon_{\mathrm{r}}$. This capacity is expressed by Eq. (5) shown in [28].

$$
C=\frac{\varepsilon_{0} \varepsilon_{r}}{d} S
$$

The discharge voltage of the $\mathrm{SC}$ is like that of an $\mathrm{RC}$, it is given by Eq. (6), as it is described in [28].

$$
V_{s c}(t)=V_{i} e^{\left(-\frac{t}{R C}\right)}
$$

where, $V_{s c}$ is the $\mathrm{SC}$ voltage and $V_{i}$ is the initial voltage. The parallel combination and/or in series of SCs allows to have the necessary voltage required by the load. In this case, the total resistance $R_{\text {total }}$ and the total capacity $C_{\text {total }}$ are given by Eq. (7) and Eq. (8) [28].

$$
\begin{aligned}
R_{\text {total }} & =n_{s} \frac{E S R}{n_{p}} \\
C_{\text {total }} & =n_{p} \frac{C}{n_{s}}
\end{aligned}
$$

where, $n_{p}$ and $n_{s}$ are numbers of SCs respectively in parallel and in series and ESR is equivalent series resistance.

The stat of charge $S O C_{i}$ of the battery and $\mathrm{SC}$ can be calculated by Eq. (9).

$$
S O C_{i}=\frac{Q_{i}(t)}{Q_{\text {ref }}} ; i=\{\text { bat }, S C\}
$$

where, $Q i$ is the charge at instant t of source i and $Q_{\text {ref }}$ is the nominal capacity of the storage element. The specifications of the battery and the SC used in this document are presented in Table 1.

Table 1. Battery, SC and PV specifications

\begin{tabular}{ccc}
\hline & Parameters & Value \\
\hline & Capacity & $50 \mathrm{AH}$ \\
& Voltage & $48 \mathrm{~V}$ \\
battery (Shenzhen O'CEL & Standard Charge & $25 \mathrm{~A}$ \\
IFM 48-500E3) [29] & Current & $30 \mathrm{~A}$ \\
& Max. Continuous & \\
& Discharge Current \\
& & \\
& Discharge current (max. & $35 \mathrm{~A}$ \\
& 2 sec. duration) & \\
RC (BCAP0100 T07 & Rated Capacitance & $2.7 \mathrm{~F}$ \\
specifications [30] & Rated Voltage & $2.7 \mathrm{~V}$ \\
& Absolute Maximum & $36 \mathrm{~A}$ \\
& Current & 18 \\
& Series branch & 6 \\
& Parallel branch & \\
& Nominal power & $1000 \mathrm{~W}$ \\
PV & Module power & $250 \mathrm{~W}$ \\
& Module number & 4 \\
& MPP tracking number & 4 \\
\hline
\end{tabular}

\subsection{DC machine, dynamic design and propulsion system}

The DC machine ensures a bidirectional power conversion. In the first direction, during the traction cycle, it converts electrical energy produced by the multi-source system into mechanical energy to feed the propulsion system. In the second direction which is corresponding to the braking phase, it converts mechanical energy into an electrical one which is stored in a storage element. In this work, the regenerative braking is not considered.

A good aerodynamic optimization could be achieved by minimizing the frontal area in order to limit the effect of the air friction force. The wheels are also finely selected to reduce the friction force with the sol. The slope friction force must be also considered.

The propulsion system transfers the torque provided by the motor to the wheel. The geometric and the dynamic characteristics of the vehicle are presented in Table 2.

Table 2. Vehicle model parameters

\begin{tabular}{cc}
\hline Parameters & Value \\
\hline Power DC machine & $1 \mathrm{~kW}$ \\
Rated voltage & $60 \mathrm{~V}$ \\
Drag coefficient, $\mathrm{C}_{\mathrm{d}}$ & 0.75 \\
Air-density & $1.25 \mathrm{~kg} \mathrm{~m}^{-3}$ \\
Frontal area, A & $0.6 \mathrm{~m}^{2}$ \\
Wheels number & 3 \\
Gear ratio, G & 2 \\
Gravity Acceleration, g & $9.81 \mathrm{~N} / \mathrm{kg}$ \\
Vehicle mass without passengers & $120 \mathrm{~kg}$ \\
\hline
\end{tabular}

\section{PROPOSED ENERGY MANAGEMENT STRATEGY}

The proposed EMS aims to maintain the stable operation of the system under any conditions taking into account the role of each source and their constraints. The implemented strategy in the EMS is based on the following criteria: 
- Criterion 1: The $S O C_{b a t}$ must operate in proper range defined by Eq (10).

$$
\operatorname{soc}_{\text {bat }}^{\min } \leq \operatorname{soc}_{\text {bat }} \leq \operatorname{soc}_{\text {bat }}^{\max }
$$

where, the $s o c_{b a t}^{\min }$ and $s o c_{\text {bat }}^{\max }$ are respectively the lower and the higher value of the $S O C_{b a t}$.

- Criterion 2: The continuous feeding the powertrain request by transferring the response to high peak power to the SC.

- Criterion 3: The keeping of the $S O C_{s c}$ in an appropriate level.

- Criterion 4: The optimization of the power trajectory in order to avoid power losses in the converters to the most possible extreme.

The priority of these criteria is considered as follows: The first criterion has priority over all other criteria; indeed, the battery ensures the power balance between system components. Then, the second criterion, the ultimate goal of the energy management strategy, comes in second order. Lastly, the third and the fourth criteria exchange the priority according to $S O C_{s c}$ level. Indeed, if the $S O C_{s c}$ is very low or very high the priority is given to the third, Otherwise the priority is devoted to the fourth.

Based on these criteria and on their priority, the operation of the multi sources system is categorized into several operation modes which are identified by four parameters: the $S O C_{b a t}$, the $S O C_{s c}$, the $P_{p v}$ and the $P_{l o a d}$. Therefore, a switching between different modes can be achieved through changes in these parameters.

Operation modes are described as follows:

$>$ Mode I: operates during high peak power demand, especially during the acceleration or high slope. In this case, the sources provide power to the powertrain system by transferring the response to the $\mathrm{SC}$ in order to avoid source overloading in the charge and the discharge cycles and to restrain high frequency power fluctuations of the battery.

$>$ Mode II: operates if:

- There is no high-power demand (the power provided by storage system is under the battery maximum power).

- One of the two following cases takes place: when the $P_{p v}$ is less than the $P_{l o a d}$ and $S O C_{s c}$ is below the optimal $S O C_{s c}\left(S O C_{s c o p t}\right)$, or when the photovoltaic production is higher than the load power and $S O C_{s c}$ is over the SOC scopt $_{\text {. }}$

$>$ Mode III: operates if:

- The $S O C_{s c}$ is in its optimal range (the considered operating ranges will be discussed in section 4.1).

- The system acts under one of the two following cases; the first is when the $P_{p v}$ is higher than the $P_{\text {load }}$ and the

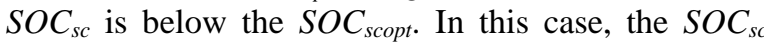
reaches the $S O C_{\text {scopt }}$ by absorbing the excess energy in the DC bus. The second is when $P_{p v}$ is lower than the $P_{\text {load }}$ and the $S O C_{s c}$ is over the SOCscopt, in this case, the $S O C_{s c}$ reaches the $S O C_{s c o p t}$ by providing the $\mathrm{DC}$ bus missing power.

> Mode IV: operates when the $S O C_{s c}$ is in the lower or upper critical range. In both cases, a rapid charge or discharge of the SC is made. Indeed, the SC is rapidly charged by the power supplied by the PV source and the power provided by the battery. However, it is quickly discharged by supplying both propulsion system and battery.

$>$ Mode V: operates when the $S O C_{b a t}$ value reaches its minimum or maximum value. For the first, the vehicle is turned off and the battery is charged through the PV generator. For the second, The PV generator is deactivated and the SC and battery feed the propulsion system.

The Figure 2 shows the power flow of mode I while the Table 3 resumes the operation state of each source for other modes.

As shown in the Figure 3, the proposed EMS acts on the power system in three hierarchical levels. The high level defines dynamically the operation mode according to system situation to ensure a good power sharing between sources taking into account their constraints. The medium level, taking in consideration the mode selected, computes reference power for the SC and the battery. The low level ensures the execution of the different actions of the superior levels. It consists of bidirectional which are controlled by PI controllers delivering the d signals DC-DC converters.

\subsection{High level design}

This level implements a fuzzy logic controller (FLC) with three input parameters, the first noted $\Delta P_{E}$ with $\Delta P_{E}=P_{l o a d}-P_{p v}$, and the second is the $S O C_{b a t}$, while the third is $S O C_{s c E}$ which represents $S O C_{s c}$ domain. While five outputs are considered for this FLC.

The Table 4 shows the universe of the fuzzy variables which are defined according to system parameters, constraints related to each source and EMS goals, Eq. (2) and Eq. (10). In the last, these variables are normalized to be used in FLC.

The membership functions of these variables are presented in Figure 4, where their intervals are designed based on EMS goals. For the input $\Delta \mathrm{P}_{\mathrm{E}}$, four membership functions are defined where high positive, high negative functions are defined to characterize high power demand operation. Nevertheless, the membership functions low negative and low positive are chosen when the vehicle operates during low power demand phase.

For the input $S O C_{b a t}$, the membership functions low and high are respectively designed to avoid surcharge and depth of discharge of the battery. However, the function meduim represents normal operation of the battery.

Five functions are considered for the variable $S O C_{s c E}$. The functions very low and very high represent the critical lower and upper areas of $S O C_{s c}$. What's more, optimal membership function is defined for optimal operating rang.

Table 3. Different operation state of sources according to operation modes

\begin{tabular}{|c|c|c|c|c|}
\hline & & SC & bat & PV \\
\hline Mode I & & Discharge & Discharge & MPP \\
\hline \multirow{2}{*}{ Mode II } & Case 1 & Charge & Discharge & \multirow{2}{*}{ MPP } \\
\hline & Case 2 & Discharge & Charge & \\
\hline \multirow{2}{*}{ Mode III } & Case 1 & Charge & - & \multirow{2}{*}{ MPP } \\
\hline & Case 2 & Discharge & - & \\
\hline \multirow{2}{*}{ Mode IV } & Case 1 & Discharge & Charge & \multirow{2}{*}{ MPP } \\
\hline & Case 2 & Charge & Discharge & \\
\hline \multirow{2}{*}{ Mode V } & Case 1 & - & Charge & \multirow[t]{2}{*}{-} \\
\hline & Case 2 & Discharge & Discharge & \\
\hline
\end{tabular}




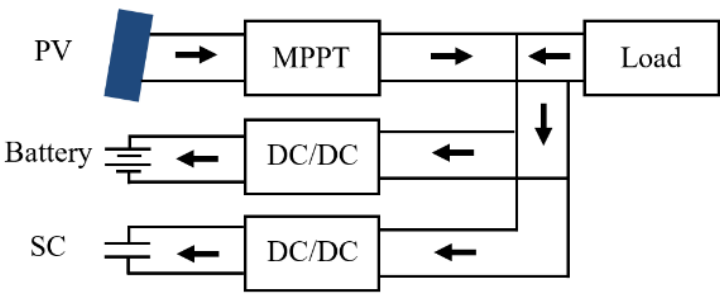

Figure 2. Power flow of mode I

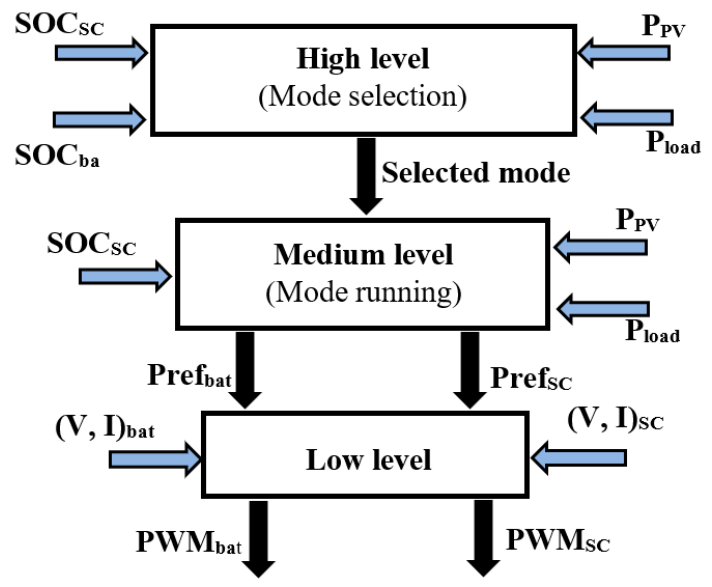

Figure 3. Architecture of the proposed hierarchical level EMS

Table 4. Universe of fuzzy variales

\begin{tabular}{ccc}
\hline & Universe & Normalized value \\
\hline $\boldsymbol{S O C}_{\text {bat }}$ & {$[0100 \%]$} & {$[01]$} \\
$\boldsymbol{S O C}_{\boldsymbol{s c}}$ & {$[0100 \%]$} & {$[01]$} \\
$\boldsymbol{A P}_{\boldsymbol{E}}$ & {$[-7000 \mathrm{~W} 7000 \mathrm{~W}]$} & {$[01]$} \\
Outputs & $\{0,1\}$ & $\{0,1\}$ \\
\hline
\end{tabular}

Five output variables are considered for this controller each with two membership functions on and off as seeing in (Figure 5). Each output represents the state of one mode, if the chosen function is on membership; the corresponding mode is activated, and is deactivated if the chosen function is off. Firstly, these variables are first fuzzified by (min-max) method. Then, the rules that relate inputs to outputs are defined using the standard Mamdani structure "if-then". Table 5 resumes the principal rules implemented in this FLC where it can be verified that the rules are in line with EMS goals. Finally, The SOM method is used for defuzzification process and converting linguistic terms of fuzzy sets to numerical value.
At each instant, one mode out of five is activated; the states of outputs are characterized by Eq. (11).

$$
\sum_{j=1}^{5} w_{j}=1, w_{j} \in\{0,1\} \quad \forall t
$$

where, $w_{j}$ represents the numerical value of the output $j$.

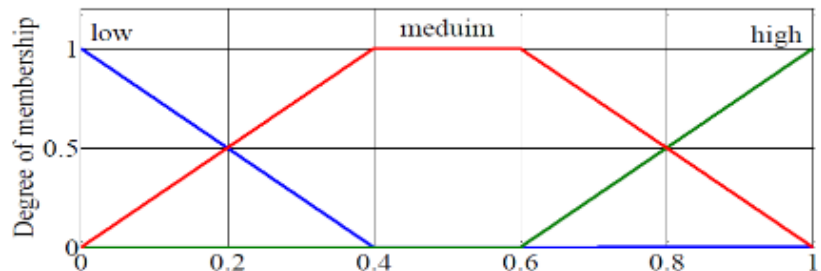

(a)

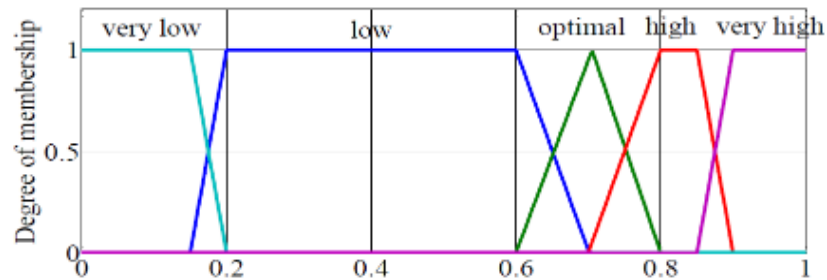

(b)

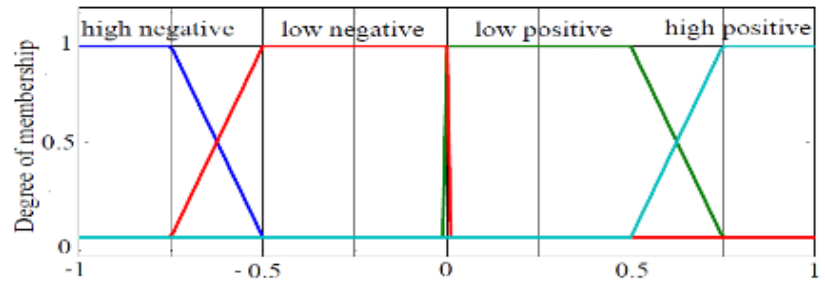

(c)

Figure 4. Normalized membership functions for inputs for high level (a) Membership functions for $S O C_{\text {bat }}$. (b)

Membership functions for $S O C_{s c}$, (c) Membership functions for $\triangle P_{E}$

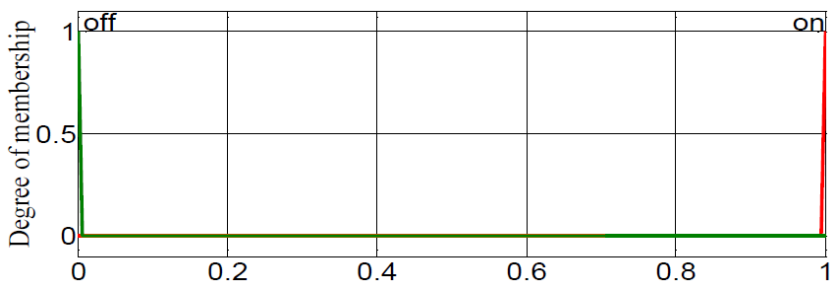

Figure 5. Normalized membership functions for outputs for high level

Table 5. FLC rules

\begin{tabular}{|c|c|c|c|c|c|c|}
\hline \multicolumn{3}{|c|}{$S O C_{b a t}$} & \multicolumn{2}{|l|}{$S O C_{s c}$} & $\Delta P_{E}$ & Activated modes \\
\hline \multirow{10}{*}{$I F$} & Not (low) & & Not (very low) & & high positive & I \\
\hline & Not (high) & & Not (very low) & & high negative & I \\
\hline & Not (low) & & low / very low & & low positive & II \\
\hline & Not (high) & & high / very high & & low negative & II \\
\hline & Not (low) & AND & high / very high & AND & low positive & III \\
\hline & Not (high) & $A N D$ & low / very low & AIVD & low negative & THEN \\
\hline & Not (low) & & very low & & high positive & IV \\
\hline & Not (high) & & very high & & high negative & IV \\
\hline & Low & & None & & None & V \\
\hline & High & & None & & high negative/low negative & $\mathrm{I} / \mathrm{V}$ \\
\hline
\end{tabular}




\subsection{Medium level design}

This level integrates an algorithm to compute the reference power for the $S C$ and the battery according to the mode selected by high level. The level uses the $S O C_{s c}$, the $P_{p v}$ and the $P_{\text {load }}$. These parameters are employed to define two sets $S O C_{s c p}$ which represent $S O C_{s c}$ domain and $\triangle P_{P}$. Two fuzzy subsets for the $S O C_{\text {scp }}$ and four fuzzy subsets for the $\Delta \mathrm{P}_{\mathrm{P}}$ were considered as seeing in Figure 6. The grade of membership $a_{i}$ with $i=\{1, \ldots, 4\}$ is respectively defined for membership of high negative, low negative, low positive and high positive of the $\Delta P_{P}$ set and $b_{i}$ with $i=\{1,2\}$ is respectively defined for membership Low and High of SOC $C_{s c p}$ set.

The reference powers of both the battery and the SC at iteration $\mathrm{k}$ can be expressed as flow Eq. (12):

$$
\operatorname{Pref}_{i\{b a t, s c\}}(k)=\sum_{j=1}^{5} w_{j}(k) \operatorname{Pref}_{i_{j}}(k) \quad \forall k
$$

where, $\operatorname{Pref} f_{i_{j}}(k)$ is the reference power of the source $i$ of the mode $\mathrm{j}$.

An algorithm which is based on fuzzy sets theory and human expertise, is developed to calculate the $P r e f_{s c}$ and Pref $_{\text {bat }}$. The pseudo code, given in appendix, has been implemented in Matlab.

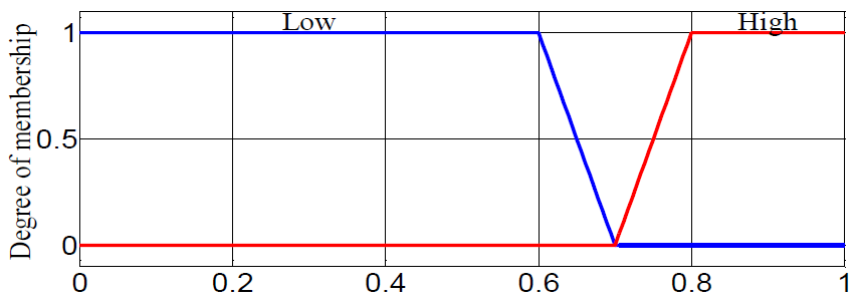

(a)

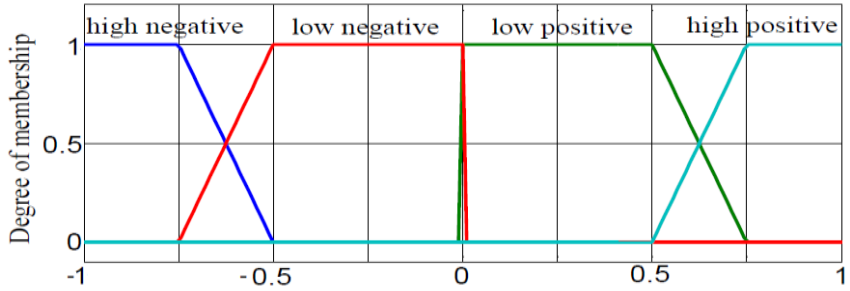

(b)

Figure 6. Normalized membership functions for medium level. (a) Membership functions for $S O C_{s c p}$. (b) Membership functions for $\triangle \mathrm{P}_{\mathrm{P}}$

\subsection{Low level design}

In this level, the PI controllers are used to ensure the Pref fat and Pref $_{s c}$ defined by the medium level. This is done by delivering PWM signal for DC/DC converters for battery and SC. While, the PV converters are controlled by $\mathrm{P} \& \mathrm{O}$ algorithm to extract the maximum available power from $\mathrm{PV}$ array. Figure 7 presents control diagram of the employed DC/DC converters.

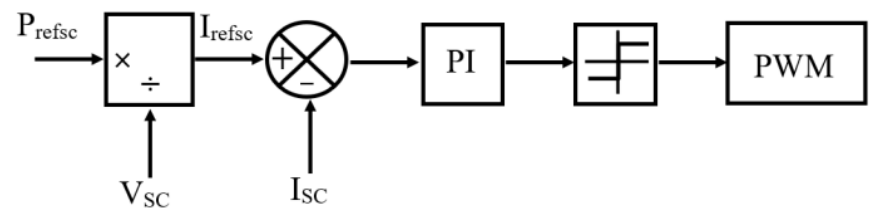

(a)

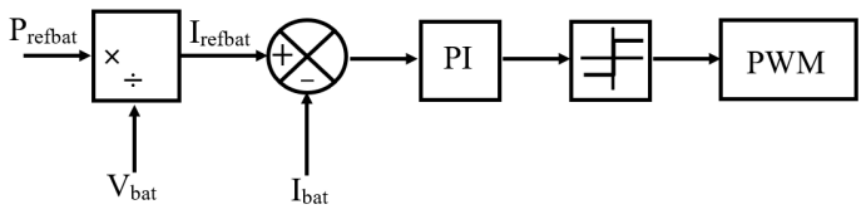

(b)

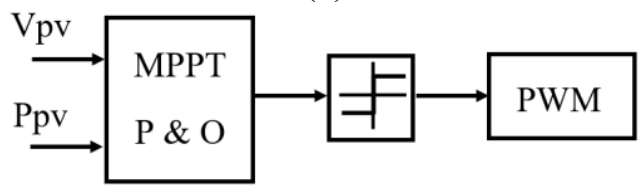

(c)

Figure 7. Control diagram of the used converters of: (a) $S C$; (b) battery; (c) $P_{p v}$ array

\section{RESULTS AND DISCUSSIONS}

The system presented above is modeled and simulated in Matlab R2013a SimPowerSystem on an Intel ${ }^{\circledR}$ CoreTMi7$3770 \mathrm{CPU}$ at $3.40 \mathrm{GHz}$ with 8 Gbytes in RAM and based on vehicle parameters which are given in Tables 1 and 2 . Different simulations are done to prove the effectiveness of the proposed EMS. For, the study vehicle is tested using two driving cycles. The first is noted A which is shown in Figure 8(a), while the second is noted B and is presented in Figure 9(a). As shown in these Figures, a driving cycle is speed-time sequence; it represents different traffic conditions [19]. The Figures 8 (b) and 9 (b) represent respectively the power demand for the two driving cycles $\mathrm{A}$ and $\mathrm{B}$.

The Figure 8 (b) shows that the power demand has a value of $7 \mathrm{~kW}$ at time $35 \mathrm{~s}$. That requires taking into account the photovoltaic generation which is about $1 \mathrm{~kW}$, the battery must provide a current of $125 \mathrm{~A}$ which goes beyond the maximum continuous current of the battery used for this vehicle (Table 1). However, the considered SC offers current up to $216 \mathrm{~A}$ at the same voltage of $48 \mathrm{v}$ which can justify the use of SC.

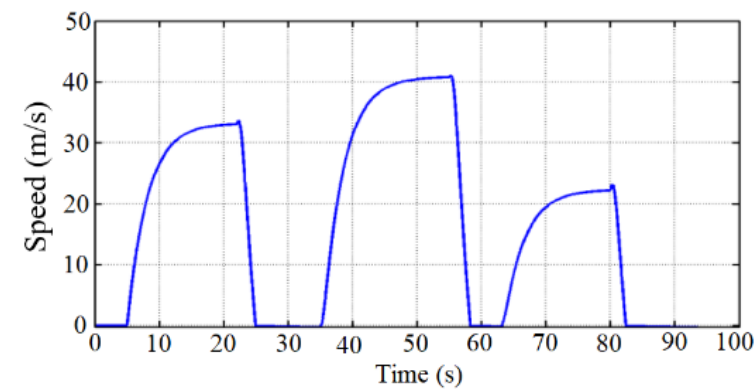

(a)

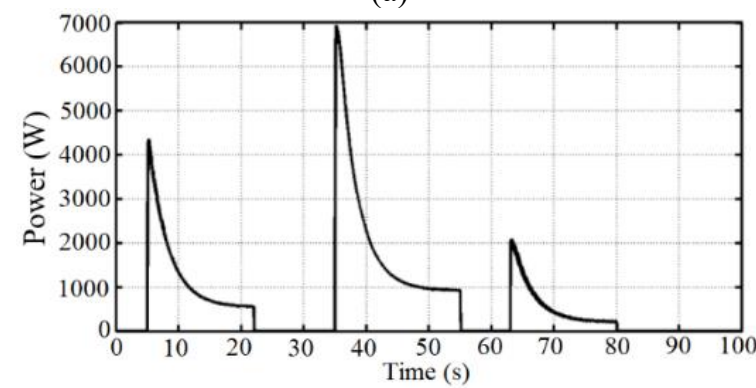

(b)

Figure 8. Different curves for driving cycle A. (a) Speed variation versus time; (b) Power demand verus time 


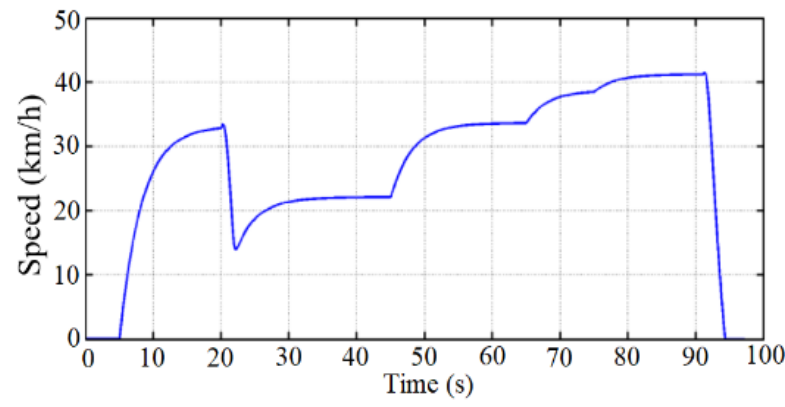

(a)

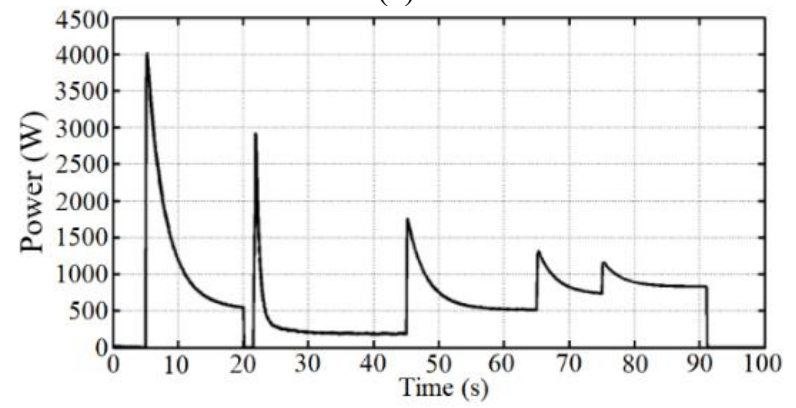

(b)

Figure 9. Different curves for driving cycle B. (a) Speed variation versus time; (b)Power demand verus time

The Figures 10 and 11 represent respectively different simulation results for driving cycle A with initial values of $S O C_{s c} ; S O C_{\text {scinit }}=100 \%$ and $S O C_{\text {scinit }}=20 \%$ which are chosen as superior and inferior critical values respectively. This allows validate the EMS and takes the system under proof. However, the battery operates at its normal condition $\left(S O C_{b a t}=50 \%\right)$, which means that the first criterion is always ensured. The results are discussed by analyzing the EMS behavior, the power sharing between sources and the respect of the priority of criteria.

The Figure 10 represents the results for driving cycle (A) with $S O C_{\text {scinit }}=100 \%$ which means that the $S O C_{s c}$ is in the superior critical range. In the first time the $\Delta \mathrm{P}_{\mathrm{E}}$ is negative, therefore the system starts with mode IV where the energy is transferred from the SC to the battery. An acceleration appears at 5 swhich is corresponding to a high-power demand. In this situation, the system moves to mode I where the load is totally supplied by the PV generator and the SC. After that, the $\Delta \mathrm{P}_{\mathrm{E}}$ becomes low positive (is below the batteries maximum power and is positive) and the $S O C_{s c}$ is still above its optimal value (high), for this reason, the system moves to mode III where the $\mathrm{SC}$ is discharged feeding the load until the $\Delta \mathrm{P}_{\mathrm{E}}$ becomes low negative, In these conditions, the system acts under mode II where energy exchange between the SC and the battery occurs, especially from the $\mathrm{SC}$ to the battery. At $22 \mathrm{~s}$, the vehicle turns off and the $S O C_{s c}$ is near of its optimal value $S O C_{\text {scopt }}$, therefore all power produced by $\mathrm{PV}$ generator is stored in the battery. Another strong acceleration is produced at time $35 \mathrm{~s}$, thanks to EMS, the system operates in mode I. After this acceleration, the $S O C_{s c}$ becomes below the $S O C_{s c o p t}$ and the power is transferred from the battery to the SC until time $55 \mathrm{~s}$ where $S O C_{s c}$ reaches the $S O C_{\text {scopt. }}$. During the third displacement and at constant speed, the battery ensures the balance by absorbing of excess energy in the DC bus.

The Figure 11 displays the results for driving cycle (A) with $S O C_{\text {scinit }}=20 \%$ that means the $S O C_{s c}$ starts with very low membership function (inferior critical area). Firstly, the vehicle is standing and no power is consumed. The EMS makes the power system operate in mode IV. During this mode, the SC is rapidly charged by power produced by the PV generator and the battery power. The same behavior of the EMS during the acceleration is observed. Specifically, the SC intervenes to support the peak demand. In constant speed, the $P_{\text {load }}$ decreases and the $\Delta \mathrm{P}_{\mathrm{E}}$ becomes low positive and the $S O C_{s c}$ is below $70 \%$.

In these conditions and in order to improve the $S O C_{s c}$ and thanks to the EMS, the system operates in mode II where the battery provides its maximum power, a part of it assists the power produced by PV generator to feed the vehicle and the other is transferred to the SC. for the second acceleration, the same cycle is repeated again (mode I): the SC and the PV generator provide the power to the load until the $\Delta \mathrm{P}_{\mathrm{E}}$ becomes low positive, as a result, the system resumes the mode II until $47 \mathrm{~s}$ where it moves to mode III. The third displacement is characterized by low power demand at constant speed. At this moment, the $S O C_{s c}$ near of its optimal value and the power produced by the PV generator is higher than the $P_{\text {load }}$. Thus, the excess of the existing energy in the DC bus is firstly sent to the SC which reaches $S O C_{\text {scopt }}=70 \%$ at $80 \mathrm{~s}$, then to the battery.

The Figures 12, 13 and 14 show the results of the cycle (B). This cycle is used to prove the effectiveness of the EMS where the speed suffers more or less important variations. The initial values that are considered for $S O C_{\text {scinit }}$ are $20 \%, 70 \%$ and $100 \%$

Thus, these Figures show the power sharing between the sources and the evolution of the $S O C_{s c}$ for various initial values. The behavior of the EMS does not differ much from the cycle A. However, some comments can be added. During strong accelerations, the SC and the PV supply the load. While, in case of low acceleration where the value of $\Delta \mathrm{P}_{\mathrm{E}}$ is below the battery maximum power, only the battery intervenes to ensure a balance between the load and the PV.

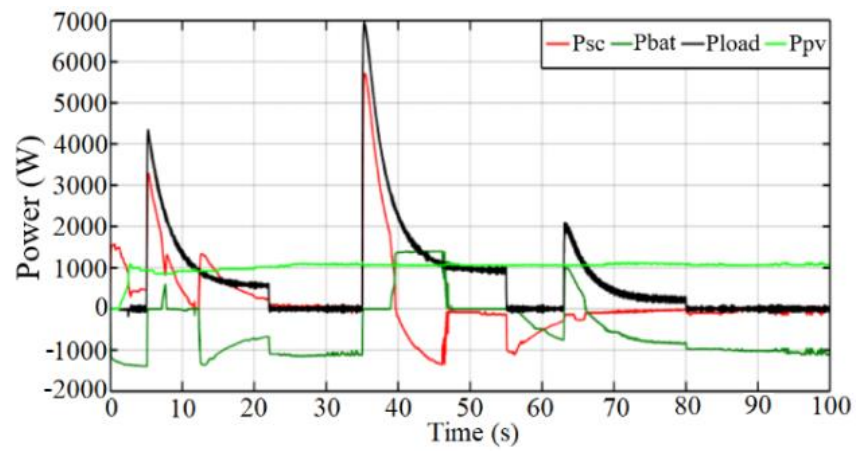

(a)

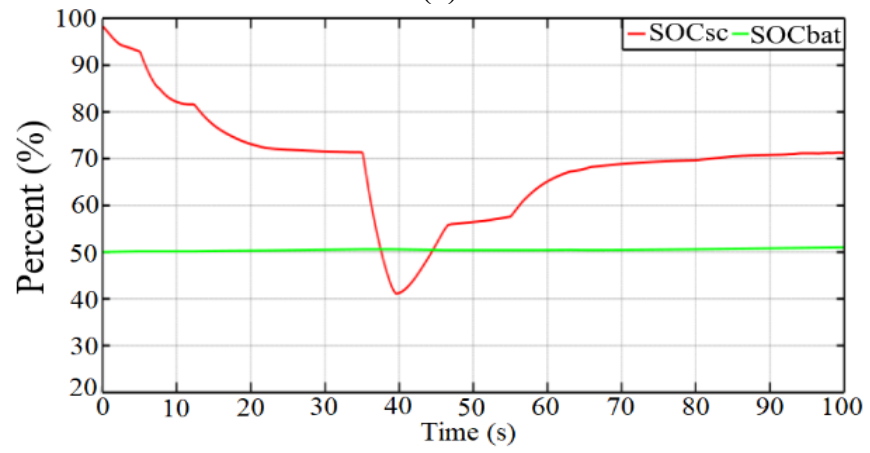

(b)

Figure 10. Simulation driving cycle A results when $S O C_{\text {scinit }}=100 \%$. (a) Power sharing verus time; (b) SOC evolution versus time for SC and battery 


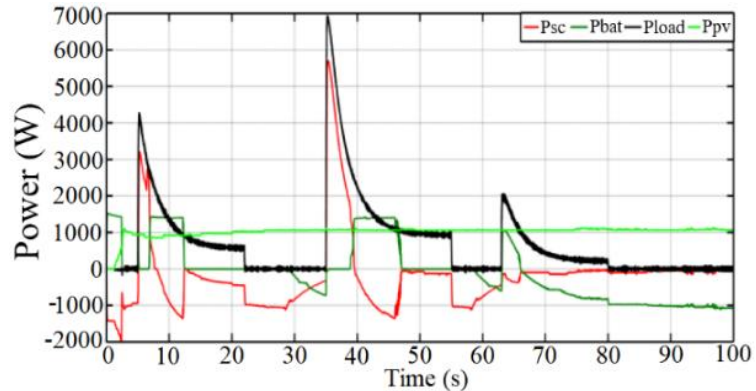

(a)

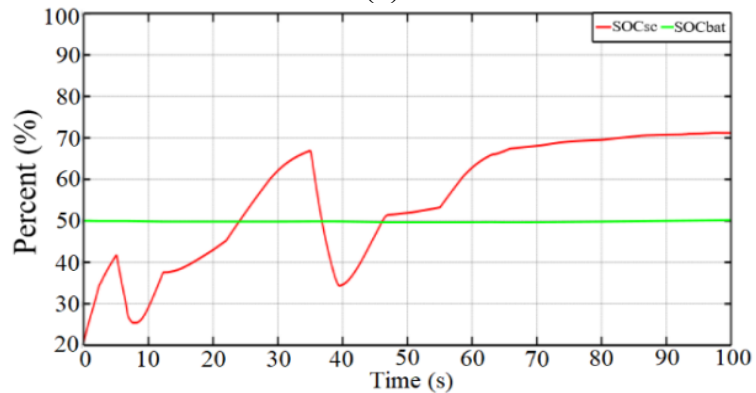

(b)

Figure 11. Simulation driving cycle A results when $S O C_{\text {scinit }}=20 \%$. (a) Power sharing verus time; (b) SOC evolution versus time for SC and battery

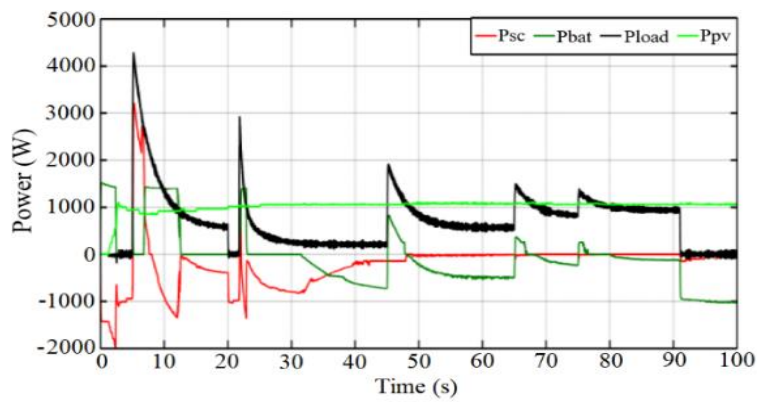

(a)

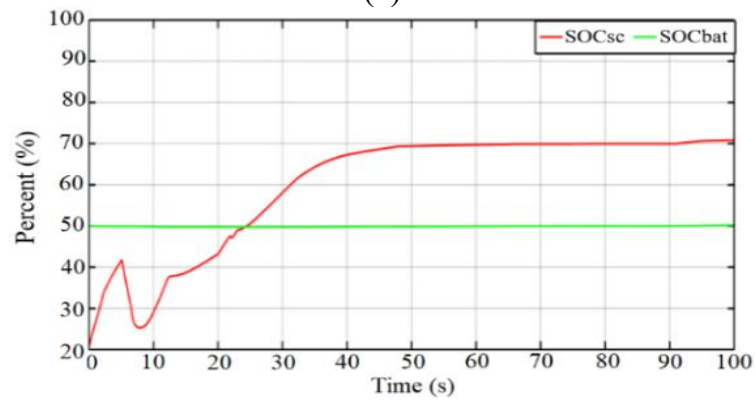

(b)

Figure 12. Simulation driving cycle $B$ results when $S O C_{\text {scinit }}=20 \%$. (a) Power sharing verus time; (b) SOC evolution versus time for SC and battery

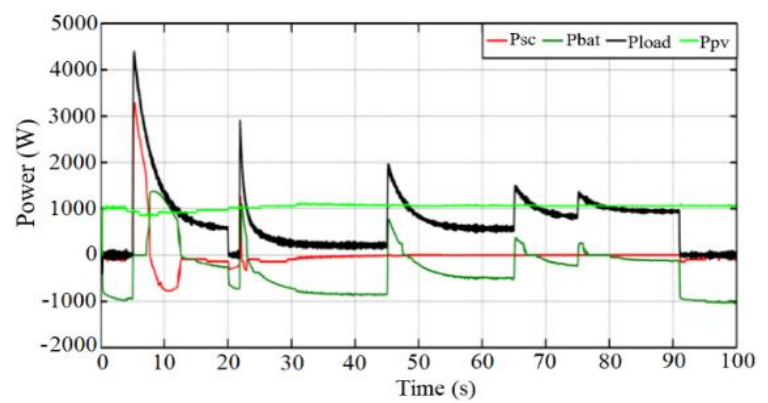

(a)

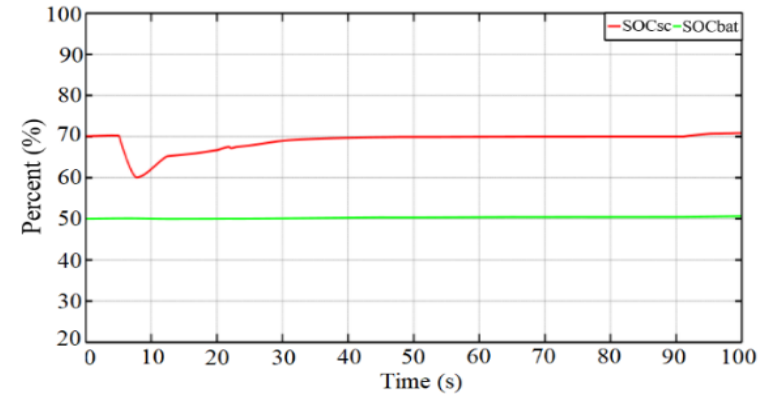

(b)

Figure 13. Simulation driving cycle $B$ results when $S O C_{\text {scinit }}=70 \%$. (a) Power sharing verus time; (b) SOC evolution versus time for SC and battery

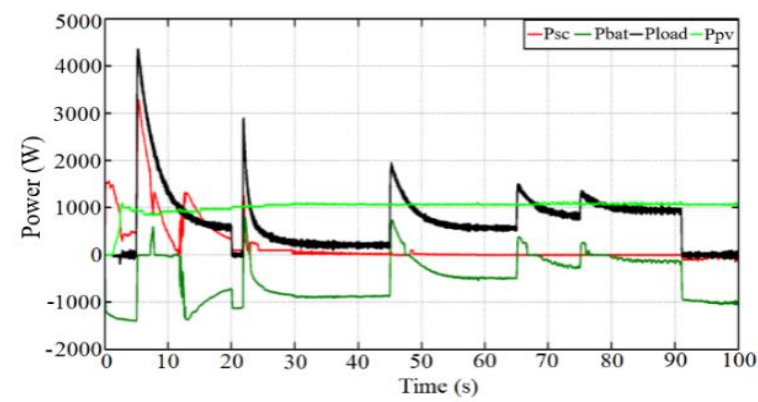

(a)

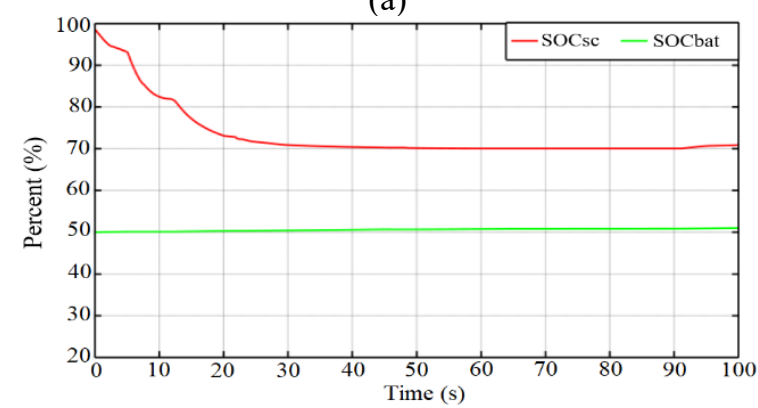

(b)

Figure 14. Simulation driving cycle $B$ results when $S O C_{\text {scinit }}=100 \%$. (a) Power sharing verus time; (b) SOC evolution versus time for SC and battery

Finally, all results presented in this paper are in accordance with the expected behavior of the developed EMS which can prove the effectiveness and reliability of energy management strategy proposed in this work.

\section{CONCLUSIONS}

In this paper, a multi-criteria EMS for an LSV which multisource system consists of a PV source and a battery and a SC has been presented. In this approach a three-hierarchical level management scheme has been formulated based on a strategy and an action planning level.

The overall system has been simulated and evaluated using the two driving cycles with different initial conditions and the results are presented. The obtained results show a high accordance and agreement with the expected behavior of the suggested EMS. These results permit to prove the efficiency and reliability of the proposed methodological approach in this paper.

Also, in the proposed architecture, integrating the $\mathrm{SC}$ in solar vehicle allows reducing the battery and $\mathrm{DC} / \mathrm{DC}$ 
converters sizing, accordingly minimizing the power losses and improving vehicle performances. In the other hand, terms of the dynamic behavior, using the present sources structure permits reducing battery stresses by providing or absorbing peaks currents.

In the last, the developed methodology in this work presents a contribution to achieve good quality and practical solutions for developing solar electrical vehicle equipped by multisources with improved range and extended battery life.

\section{REFERENCES}

[1] Hartmann, B. (2020). Comparing various solar irradiance cetegorization methods - a critique on robustness. Renewable Energy, 154: 661-671. https://doi.org/10.1016/j.renene.2020.03.055

[2] Muneer, T., Kolhe, M., Doyle, A. (2017). Electric Vehicles: Prospects and Challenges. Elsevier, ISBN: 978-0-12-803021-9. https://doi.org/10.1016/C2014-004033-6

[3] Fathabadi, H. (2017). Novel solar powered electric vehicle charging station with the capability of vehicle-togrid. Solar Energy, 142: 136-143. https://doi.org/10.1016/j.solener.2016.11.037

[4] Belattar, A., Chennani, M., Doubabi, S. (2015). TS fuzzy control for dc-dc boost converter in PV systems. In: Proceedings of the IEEE 3rd International Renewable and Sustainable Energy Conference Marrakesh, Morroco. https://doi.org/10.1109/IRSEC.2015.7455051

[5] Sun, K., Zhang, L., Xing, Y., Guerrero, J.M. (2011). A distributed control strategy based on DC bus signaling for modular photovoltaic generation systems with battery energy. IEEE Transactions on Power Electronics, 26(10): 3032-3045. https://doi.org/10.1109/TPEL.2011.2127488

[6] Dahbi, M., Doubabi, S., Rachid, A. (2015). Autonomy analysis of a solar electric tricycle. In: Proceedings of the IEEE 3rd International Renewable and Sustainable Energy Conference Marrakesh, Morroco. https://doi.org/10.1109/IRSEC.2015.7454954

[7] Gagliardi, F., Pagano, M., Maestri, G., Martone, M., Tarantino, A. (2002). Experimental results of on-board battery-ultracapacitor system for electric vehicle applications. In: Proceedings of the IEEE International Symposium on Industrial Electronics, Aquila, Italy, 1: 93-98. https://doi.org/10.1109/ISIE.2002.1026047

[8] Pay, S., Baghzouz, Y. (2003). Effectiveness of batterysuper capacitor combination in electric vehicles. In: Proceedings of the IEEE Power Tech Conference, Bologna, https://doi.org/10.1109/PTC.2003.1304472

[9] Yang, Y.P., Liu, J.J., Hu, T.H. (2011). An energy management system for a directly-driven electric scooter. Energy Conversion and Management, 52(1): 621-629. https://doi.org/10.1016/j.enconman.2010.07.038

[10] Onar, O.C., Uzunglu, M., Alam, M.S. (2006). Dynamic modeling, design and simulation of a wind/fuel cell/ultracapacitor-based hybrid power generation system. Journal of Power Sources, 161(1): 707-722. https://doi.org/10.1016/j.jpowsour.2006.03.055

[11] Belhadj, S., Belmokhtar, K., Ghedamsi, K. (2019). Improvement of energy management control strategy of fuel cell hybrid electric vehicles based on artificial intelligence techniques. Journal Européen des Systèmes
Automatisés,

52(6):

$541-550$

https://doi.org/10.18280/jesa.520601

[12] Zhang, H.T., Qin, Y.Z., Li, X.G., Liu, X.Z., Yan, J.Y. (2020). Power management optimization in plug-in hybrid electric ehicles subject to uncertian driving cycles. eTransportation, 3: 100029. https://doi.org/10.1016/j.etran.2019.100029

[13] Chacko, P.J., Sachidananda, M. (2020). Optimization \& validation of intelligent energy management systemfor pseudo dynamic predictive regulation of plug-inhybrid electric vehicle as donor clients. eTransportation, 3: 100050. https://doi.org/10.1016/j.etran.2020.100050

[14] Morsalin, S., Haque, A., Mahmud, A. (2020). Machine to machine performance evaluation of grid-integrated electric vehicles by using various scheduling algorithms. eTransportation, 3:

100044. https://doi.org/10.1016/j.etran.2020.100044

[15] Song, S.X., Sun, W.C., Xiao, F., Peng, S.L., An, J.Y., Wang, D. (2017). A novel coordinated control algorithm for distributed driving electric vehicles. Journal Européen des Systèmes Automatisés, 50(4-6): 405-421. https://doi.org/10.3166/JESA.50.405-421

[16] Hannan, M.A., Azidin, F.A., Mohamed, A. (2012). Multi-sources model and control algorithm of an energy management system for light electric vehicles. Energy Conversion and Management, 62: 123-130. https://doi.org/10.1016/j.enconman.2012.04.001

[17] Neffati, A., Guemri, M., Caux, S., Fadel, M. (2013). Energy management strategies for multi source systems. Electric Power Systems Research, 102: 42-49. https://doi.org/10.1016/j.epsr.2013.03.008

[18] Ustun, O., Yilmaz, M., Gokce, C., Karakaya, U., Tuncay, R.N. (2009). Energy management method for solar race car design and application. In: Proceedings of the IEEE International Electric Mach Drives Conference, Miami, USA pp. 804-811. https://doi.org/10.1109/IEMDC.2009.5075296

[19] Trovao, J.P., Pereirinha, P.G., Jorge, H.M., Antunes, C.H (2013). A multi-level energy management system for multi-source electric vehicles - an integrated rule-based meta-heuristic approach. Appied Energy, 105: 304-318. https://doi.org/10.1016/j.apenergy.2012.12.081

[20] Rosario, L.C., Luk, P.C.K. (2006). Implementation of a modular power and energy management structure for battery - ultracapacitor powered electric vehicles. In: Proceedings of the IET Hybrid Vehicle Conference, Coventry, UK, pp. 141-156. https://doi.org/10.1049/cp:20060605

[21] Ceraolo, M., Donato, A.D., Franceschi, G. (2008). A general approach to energy optimization of hybrid electric vehicles. IEEE Transactions Vehicular Technology, 57(3): 1433-1441. https://doi.org/10.1109/TVT.2007.909268

[22] Melero-Perez, A., Gao, W., Fernandez-Lozano, J.J. (2009). Fuzzy logic energy management strategy for Fuel Cell/Ultracapacitor/Battery hybrid vehicle with Multiple-Input DC/DC converter. In: Proceedings of the IEEE Vehicle Power and Propulsion Conference, Dearborn, USA, pp. 199-206. https://doi.org/10.1109/VPPC.2009.5289851

[23] Sizikov, G., Kolodny, A., Fridman, E.G., Zelikson, M. (2010). Efficiency optimization of integrated DC-DC buck converters. In: Proceedings of the $17^{\text {th }}$ IEEE International Conference on Electronics, Circuits and 
Systems, Athens, Greece, pp. 1215-1218. https://doi.org/10.1109/ICECS.2010.5724735

[24] Moroccan Solar Race, Technical rules, www.moroccansolarrace.com, accessed on 25 December, 2019.

[25] Datta, M., Senjyu, T., Yona, A., Funabashi, T. (2011). A fuzzy based method for leveling output power fluctuations of photovoltaic-diesel hybrid power system. Renewable Energy, 36(6): 1693-1703. https://doi.org/10.1016/j.renene.2010.12.009

[26] Patcharaprakiti, N., Premrudeepreechacharn, S., Sruithaisiriwong, Y. (2005). Maximum power point tracking using adaptive fuzzy logic control for gridconnected photovoltaic system. Renewable Energy, 30(11): 1771-1788. https://doi.org/10.1016/j.renene.2004.11.018

[27] Tremblay, D., Dessaint, L.A., Dekkiche, A. (2007). A generic battery model for the dynamic simulation of hybrid electric vehicles. In: Proceedings of the IEEE Vehicle Power and Propulsion Conference, Arlington, USA, $\mathrm{pp}$. 284-289. https://doi.org/10.1109/VPPC.2007.4544139

[28] Sharma, P., Bhatti, T.S. (2010). A review on electromechanical double-layer capacitors. Energy Conversion Management, 21(12): 2901-2912. https://doi.org/10.1016/j.enconman.2010.06.031

[29] O'CEL IFM 48-500E3 Datasheets (Document number: S460XLP21), Shenzhen O'CELL Technology Co., Ltd.

[30] Maxwell Technologies USA BOOSTCAP Ultracapacitor Modules Datasheet (Document number: 1013793.9), www.maxwell.com, accessed on 15 December, 2019.

\section{APPENDIX}

Do

Get mode; with $\mathrm{i}=1 \ldots .5$

Get $a_{i}, b_{j}, \mathrm{i}=1 \ldots 4$ and $\mathrm{j}=1 \ldots 2$

Get $\Delta \mathrm{P}(\mathrm{k})$

If $(\Delta \mathrm{P}(\mathrm{k})<0)$

$P_{b}=P_{b a t}^{\min }$;

Else

$P_{b}=P_{\text {bat }}^{\max }$

Switch mode

Case 1:

Pref $_{\text {sc }}=\left(\mathrm{a}_{1}+\mathrm{a}_{4}\right) \Delta \mathrm{P}(\mathrm{k})$;

Pref $_{\text {bat }}=\left(\mathrm{a}_{2}+\mathrm{a}_{3}\right) \Delta \mathrm{P}(\mathrm{k})$;

Case 2:

$\operatorname{Pref}_{\mathrm{sc}}=-\operatorname{sign}(\Delta \mathrm{P}(\mathrm{k}))\left(\mathrm{b}_{1}+\mathrm{b}_{2}\right)\left(\mathrm{P}_{\mathrm{b}}-\Delta \mathrm{P}(\mathrm{k})\right) ;$

$\operatorname{Pref}_{\mathrm{bat}}=\Delta \mathrm{P}(\mathrm{k})+\operatorname{sign}(\Delta \mathrm{P}(\mathrm{k}))\left(\mathrm{b}_{1}+\mathrm{b}_{2}\right)\left(\mathrm{P}_{\mathrm{b}}-\Delta \mathrm{P}(\mathrm{k})\right)$;

Case 3:

Pref $_{\text {sc }}=\left(\mathrm{b}_{1}+\mathrm{b}_{2}\right) \Delta \mathrm{P}(\mathrm{k})$;

Pref $_{\text {bat }}=\left(1-\left(b_{1}+b_{2}\right) \Delta P(k)\right.$;

Case 4:

$\operatorname{Pref}_{\mathrm{sc}}=\Delta \mathrm{P}(\mathrm{k})+\operatorname{sign}(\Delta \mathrm{P}(\mathrm{k})) \mathrm{P}_{\mathrm{b}}$

$\operatorname{Pref}_{\mathrm{bat}}=\operatorname{sign}(\Delta \mathrm{P}(\mathrm{k})) \mathrm{P}_{\mathrm{b}}$

Case 5:

$\operatorname{Pref}_{\mathrm{sc}}=\operatorname{sign}(\Delta \mathrm{P}(\mathrm{k}))\left(\mathrm{b}_{1}+\mathrm{b}_{2}\right) \Delta \mathrm{P}(\mathrm{k})$;

Pref $_{\text {bat }}=\mathrm{P}_{\mathrm{pv}}(\mathrm{k})$;

Break

Output $\left(\right.$ Pref $_{\mathrm{sc}}$, Pref $\left._{\text {bat }}\right)$;

End While

END 